73.

\section{Lack of Prehospital Death Pronouncement by Air Medical Programs May Waste Health Care Resources}

Bartholomew J. Tortella, MTS, MD, FACS, * Robert Simon, Robert F. Lavery, MA, MICP

New Jersey Trauma \& EMS Research Center University

Hospital, Newark, New Jersey USA

Statement of Purpose: Prehospital death pronouncement policies are common among ground ambulances. No previous report has addressed such policies among Air Medical (AM) programs.

Statement of Methods: Structured telephone interviews of 125 of 143 (87\%) AM programs (14 fixed wing, 68 rotor wing, 43 both) were conducted by research staff from 6 to $8 / 95$.

Summary of Results: Only fifty percent of AM crews are permitted to pronounce death in the field. Of those AM programs which permit pronouncement, direct medical command physician contact is required in $35 \%$ of pronouncements while $25 \%$ of programs utilize protocols. Of those AM programs which permit pronouncement, $77 \%$ of flight nurses can pronounce while only $33 \%$ of paramedics. Most programs (95\%) conduct $\mathrm{QA}$ reviews of all deaths.

Statement of Conclusions: Prehospital death pronouncement is permitted in only $50 \%$ of AM programs. Since prehospital death pronouncement may obviate the need for $\mathrm{AM}$ transport, AM programs may be transporting clinically dead but unpronounced patients wasting scarce medical resources. This differs greatly from ground EMS programs where death protocols are common. Particularly puzzling is the small percentage of flight paramedics who are allowed to pronounce when compared to flight nurses since ground EMS pronouncements are performed by paramedics. AM programs should review their prehospital pronouncement protocols since managed care initiatives will drive health care organizations to abandon the wasteful practice of transporting clinically dead, but unpronounced, patients.
74.

Location of Cardiac Arrests: Implications for AED Placement

James $M$. Atkins, $M D$, * Brian S. Zachariah, $M D$

University of Texas Southwestern Medical Center, Dallas,

Texas USA

Hypothesis: First responder organizations with automated external defibrillators (AEDs) can have a larger impact on survival of out-of-hospital cardiac arrest than placing AEDs in large buildings.

Methods: To evaluate the impact, all cardiac arrests handled by a large urban fire department for 1994 were analyzed. Each 5.6 square mile area of the city was defined as business (Bus), high (HiIRes), middle (MiIRes), or low income (LoIRes) residential. For each area, the CPR rates were calculated for the number of arrests $/ 100$ ambulance dispatches, and were stratified by percent of adults over age 65 .

Results: Of the 1,222 cardiac arrests, only 85 occurred in business and industrial areas, 1,041 occurred in residential areas. The downtown business district had only 77 arrests with half of those being outside of buildings or in shelters. The data by area is:

\begin{tabular}{|c|c|c|c|c|}
\hline & Bus & HilRes & MilRes & LoIRes \\
\hline $\begin{array}{l}\text { CPR rate/ } \\
100 \text { dispatches }\end{array}$ & 0.69 & 1.12 & 1.03 & 0.98 \\
\hline $\begin{array}{l}\text { CPR rate/ } \\
\text { square mile }\end{array}$ & 2.18 & 1.82 & 3.60 & 11.22 \\
\hline
\end{tabular}

\begin{tabular}{|c|c|c|c|c|}
\hline & $<5 \%$ & 5-15\% & $15-25 \%$ & $>25 \%$ \\
\hline $\begin{array}{l}\text { CPR rate/ } \\
100 \text { dispatches }\end{array}$ & 0.39 & 0.94 & 1.09 & 1.72 \\
\hline
\end{tabular}

Stratifying the areas by percent of adults over age 65 shows: Conclusions: High rise buildings have a high population density, but the incidence of arrests are extremely low. Most arrests occur in residential areas. The arrests/square mile are inversely related to annual income. The incidence of arrests is also dependent on the percent of adults over age 65 . Due to the logistics defined by this distribution, first responders organizations armed with AEDs and EMS systems with defibrillators will have a greater impact than placing AEDs in large buildings. 